\title{
Informelles E-Learning: Ist ein Paradigmenwechsel notwendig?
}

\author{
Eine videografische Feldanalyse der Auswirkungen digitaler Medien auf das Lernen im Alltag
}

\author{
Otto Petrovic ${ }^{1}$ \\ Online publiziert: 8. Juli 2020 \\ (c) Der/die Autor(en) 2020
}

\section{Zusammenfassung}

E-Learning ist ein bunter Strauß an Technologien und pädagogischen Konzepten, gepaart mit unterschiedlichen Formen der Wirkungsforschung. Vannevar Bushs Vision einer riesigen Erweiterung des menschlichen Gedächtnisses, Lernmanagementsysteme und Massive Open Online Courses sind allgegenwärtig. Ein großer Teil des Lernens im kognitiven, affektiven und psychomotorischen Bereich findet jedoch nebenläufig als Begleiterscheinung der Nutzung digitaler Medien im Alltag statt. Es wird noch weitgehend von Lehrenden und der E-Learning-Forschung ignoriert. Der vorliegende Beitrag bespricht zunächst markante, oftmals unhinterfragte Paradigmen des E-Learning, die zu diesen Paralleluniversen von formalem und informellem Lernen führen. Um Veränderungspotenziale digitaler Medien für das informelle Lernen zu analysieren, zeichnen 172 Lernende im Alltag 543 informelle E-Learning-Episoden als Screencasts auf, die ergänzt werden durch partizipative Feldforschung des Autors in entlegenen Regionen und Kulturkreisen. Die Schlussfolgerungen können Lehrenden helfen, das informelle Lernen ihrer Studierenden für formale Lernziele zu nutzen. Lernenden soll die Beeinflussung durch alltägliche Mediennutzung bewusst werden, um ihr Informationsverhalten kritisch zu hinterfragen.

\section{Mainstream des E-Learning}

Vor dem Hintergrund unterschiedlicher Medientheorien [31] sehen wir digitale Medien als „Mittel oder Kanäle zur Kommunikation zwischen Menschen, Menschen und Maschinen und zwischen Maschinen, die einen Wahrnehmungsraum für die Teilnehmer am Kommunikationsprozess erzeugen“. Somit sehen wir Medien nicht nur als Mittel für die Repräsentation und den Transport eines bestimmten Ausschnitts der Realität, sondern vielmehr für die Kommunikation einer bestimmten Sichtweise auf die Realität. Lernen wird als Erwerb von neuem oder geändertem Wissen sowie von Fähigkeiten, Kompetenzen und Wahrnehmungen verstanden. Informelles Lernen umfasst kognitive, affektive und psychomotorische Lernprozesse, die beiläufig bei der Durchführung anderer Tätigkeiten stattfinden. Lernen ist eine „Nebenerscheinung“ und nicht

Otto Petrovic

otto.petrovic@uni-graz.at

1 Institut für Informationswissenschaft und Wirtschaftsinformatik, Karl-Franzens-Universität Graz, Universitätsstraße 15/G3, 8010 Graz, Österreich das Ziel dieser Tätigkeiten [27]. Lehrende sehen meist ihren Aufgabenbereich im formalen Lernen. Der Beitrag soll zeigen, dass ein Verständnis für informelles Lernen und seine Berücksichtigung in formalen Settings neue Wege zur Erreichung von Lernzielen öffnet. Werden informelle Lernprozesse durch digitale Medien ermöglicht oder beeinflusst, sprechen wir von informellem E-Learning. WEIRD-Lernende steht für Lernende, die aus der Western Hemisphere stammen, Educated sind und in einem Umfeld leben, das Industrialized, Rich und Developed ist (Tab. 1).

\section{Paradigma 1: Wissenstransfer in formalen Settings}

\section{Wissenstransfer steht im Mittelpunkt}

Ein hoher Anteil an E-Learning-Technologien und -Methoden konzentriert sich auf die Unterstützung des Wissenstransfers vom Lehrenden zu Lernenden. Lehrende unterstützen ihren Vortrag mittels digitaler Medien, setzen Formen des Blended Learning zur Verknüpfung des Präsenzunterrichts mit der Nutzung digitaler Medien außerhalb des Hörsaals ein oder führen Fernunterricht durch. Gerade letzterer wird gegenwärtig oftmals als vermeintlich deutsches Synonym des Begriffs E-Learning verwendet. In der vor- 
Tab. 1 Häufig anzutreffende Paradigmen im E-Learning

Wissenstransfer vom Lehrenden zu Lernenden mittels Videos von Vorlesungen und digitalem Lehrmaterial. Wenig Berücksichtigung des Großteils des E-Learning, das beiläufig im Alltag stattfindet

Entwicklung und Nutzung dedizierter E-Learning-Technologien. Die Ubiquität digitaler Medien, vor allem des Internets der Dinge (IoT), wird noch kaum in formalen Settings genutzt

Maximieren von „,immer und überall“, Sharing und Personalisieren mit ambivalenten Auswirkungen auf fundamentale Innovationen, gesellschaftlich erwünschte Lerninhalte und soziale Anschlussfähigkeit

Annahme eines Technikdeterminismus in der Wirkungsforschung mit Vernachlässigung von soziokulturellen Rahmenbedingungen und persönlicher Entscheidungsfreiheit

Wirkungsforschung erfolgt zum überwiegenden Teil mit WEIRD-Lernenden, die besonders ungeeignet sind, um Schlussfolgerungen auf die restlichen $88 \%$ der Weltbevölkerung zu ziehen

liegenden Arbeit wird dem jedoch nicht gefolgt. Während E-Learning das gesamte Spektrum der Veränderung oder Ermöglichung von Lernprozessen durch digitale Medien umfasst, ist Fernunterricht der standortverteilte Wissenstransfer vom Lehrenden zum Lernenden. Hierzu gehört etwas auch ein im Fernsehen ausgestrahlter Lehrfilm.

Der durch digitale Technologien veränderte oder ermöglichte Wissenstransfer bedient sich des computergestützten Unterrichts in Form von lehrbuchartigen Lektionen, intelligenten Tutorsystemen sowie unterschiedlichen Arten der Organisation und Distribution von Lernmaterial, etwa in Form von Massive Open Online Courses (MOOCs). Die Befragung von 662 Lehrenden an deutschen Hochschulen zeigt eine deutliche Dominanz dieser Technologien. $92 \%$ gaben an, Präsentationstools zu nutzen, $74 \%$ digitale Texte, $59 \%$ Videoangebote und rund die Hälfte setzen Lernmanagementsysteme ein. Hingegen werden Foren, Communities und Blogs nur von $18 \%$ und soziale Netzwerke von $5 \%$ genutzt $[1,21,30]$. Die Analyse aller 142 Beiträge der CSEDU 2019 - eine der international wichtigsten Konferenzen auf dem Gebiet des E-Learning - ergab, dass auch in der Wirkungsforschung der Wissenstransfer dominiert und sich weniger als ein Fünftel der Arbeiten explizit mit dem Generieren von eigenem Wissen durch Lernende beschäftigt [5].

Diese Dominanz von Technologien zur Unterstützung des Wissenstransfers ergibt sich vor allem aus dem weitverbreiteten Selbstverständnis von Lehrenden, die „Quelle“ von Wissen zu sein. Dies gilt für Lehrende im Hörsaal genauso wie für Youtuber mit einer Million Followern. Aus lerntheoretischer Sicht liegen diesem Paradigma objektivistische Lerntheorien zugrunde, die von Wissen ausgehen, das außerhalb der Sinne des erkennenden Subjekts als letztgültige Wahrheit existiert. Mittels E-Learning wird versucht, dieses beobachtbar, quantifizierbar, strukturiert vermittelbar und kontrolliert beim Lernenden abrufbar zu machen. Für Probleme allerdings, die nicht in absolut gleicher Form im Hörsaal gelöst wurden, benötigen Lernende die Fähigkeit, gemerkte Fakten und Lösungswege neu zu arrangieren und zu deuten, um durch eigenständige Denkprozesse Lösungen zu finden. Die Notwendigkeit hierfür steigt vor allem durch Globalisierung, Wertepluralismus, Bedeutungsverlust traditioneller Autoritäten und zunehmende Geschwindigkeit des technischen Fortschritts. Hier setzen E-Learning-Systeme an, die konstruktivistischkollaboratives Lernen unterstützen. Lernende konstruieren eigenes Wissen durch Interagieren und Experimentieren mit ihrer Umwelt. Wissen wird als etwa gesehen, das sich dynamisch verändert und im sozialen Kontext ausgehandelt wird [7]. Technologien zur Unterstützung von konstruktivistisch-kollaborativem Lernen ermöglichen vor allem das Erstellen und Teilen nutzergenerierter Inhalte sowie deren Strukturierung und kritische Reflexion. Ebenso unterstützen Simulationssysteme auf Basis von Virtual Reality und Game-based Learning [23] diese Form des Lernens. Lehrende nutzen jedoch diese Technologien noch kaum [21]. Für eine ausführliche Besprechung von Lerntechnologien in Verbindung mit Lerntheorien vgl. [8, 26].

Durch digitale Medien entstehen immer mehr Gelegenheiten für konstruktivistisch-kollaboratives Lernen im Alltag. Dieses beeinflusst unmittelbar die Ziele und Ergebnisse der Lehre in formalen Settings. Etwa bleiben Fragen der Privacy im Bereich der Informatik nicht länger abstrakte Konzepte, die im späteren Berufsleben - vielleicht - Anwendung finden. Vielmehr sind Lernende im Alltag laufend damit konfrontiert und Lehrende sind nur eine von vielen Wissensquellen für den Aufbau von eigenem Wissen.

\section{Formales E-Learning dominiert}

Die Untersuchungen von $[1,21]$ zeigt weiter, dass E-Learning in den meisten Fällen zur Unterstützung des formalen Lernens im Hörsaal oder durch Selbststudium eingesetzt wird. Auch dies scheint wenig überraschend, da genau das als Aufgabe von Lehrenden angesehen wird. Wie weiter unten besprochen, können Lehrende allerdings auch das informelle Lernen berücksichtigen und zum Erreichen der Lernziele nutzen.

Der Großteil des Lernens erfolgt nicht in formalen Settings, sondern informell. Hierbei ist das primäre Ziel einer Aktivität nicht das Lernen, sondern es erfolgt beiläufig als Nebenprodukt einer anderen Aktivität. Beispiele sind etwa E-Sport, das Planen einer Reiseroute im Web oder die Nut- 
zung von Self-Measurement Devices wie Vital- und Bewegungssensoren im Sport. Informelles Lernen hat keine klaren Strukturen und im Vorhinein festgelegte Ziele, erfolgt meist in natürlicher Verknüpfung mit dem Alltagsleben und resultiert in keinem formalen Abschluss. Es umfasst Lernen im kognitiven, affektiven und psychomotorischen Bereich [4].

In OECD-Ländern verbringen 15-Jährige im Jahresdurchschnitt 2,5 h am Tag verpflichtend im Klassenzimmer, ihre Lehrenden 1,9h [22]. Bei mehrjähriger Betrachtung bleiben diese Werte weitgehend konstant. Die ARD/ZDFOnlinestudie [6] weist in der Altersgruppe der 14- bis 29Jährigen eine tägliche Internetnutzung von $5 \mathrm{~h}$ und $44 \mathrm{~min}$ aus, wobei dieser Wert von 2017 auf 2018 um $74 \mathrm{~min}$ anstieg. Hierbei nicht inkludiert ist die stark zunehmende Nutzungszeit des Internets der Dinge, etwa sprachgesteuerte Assistenten oder Smartwatches [24]. Vergleicht man die Nutzungszeit digitaler Medien im Alltag und die damit einhergehenden informellen Lernprozesse mit der Zeit des E-Learning in formalen Settings, erkennt man die starke Dominanz des informellen E-Learning innerhalb des LernÖkosystems. Der „Hebel“ für Lehrende ist hier nennenswert größer als im klassischen E-Learning.

Um dieser Argumentation zu folgen, sollte Lernen nicht nur als Erwerb von neuem oder geändertem Wissen angesehen werden, sondern auch von Fähigkeiten, Kompetenzen und Wahrnehmungen. Was hat das Spielen eines Massively Multiplayer Online Game mit Lernen von Informatikfächern zu tun? Games sind oftmals best practices für Machine Learning, Computer Vision, Artificial Intelligence und Human Computer Interaction. Hierzu kommt der Erwerb von Metakompetenzen wie Teambuilding, die nützlich in verteilten Softwareentwicklungsteams sind.

In der Begleit- und Wirkungsforschung sind empirische Arbeiten zur Beeinflussung des informellen Lernens durch digitale Medien noch selten anzutreffen. Die Untersuchung von [5] zeigt, dass sich nur 5\% der 142 analysierten Arbeiten explizit mit informellem Lernen auseinandersetzen.

Sowohl das $\boldsymbol{E}$ - (die Nutzung digitaler Medien) als auch das Learning (auf informelle Art) finden zum großen Teil außerhalb des Hörsaals statt - weitgehend entkoppelt von Lehrenden und der Wirkungsforschung.

\section{Paradigma 2: Entwicklung und Nutzung dedizierter E-Learning-Technologien}

Dedizierte E-Learning-Systeme spielen innerhalb des gesamten Lern-Ökosystems eine untergeordnete Rolle. Die seit 2007 von [9] durgeführte Befragung von 2524 Hochschullehrenden und Weiterbildungsverantwortlichen in Unternehmen aus 46 Ländern $\mathrm{zu}$ den für das Lernen bedeutendsten digitalen Medien zeigt, dass mit Moodle ein an Hochschulen gängiges dediziertes E-Learning-System erst an fünfzigster Stelle genannt wird. In der Auflistung von digitalen Medien, die von Lernenden aus eigenem Antrieb genutzt werden, erscheint es gar nicht. Den Spitzenplatz nimmt YouTube ein, auf das jede Minute $300 \mathrm{~h}$ Videomaterial hochgeladen wird und auf dem täglich fünf Milliarden Videos gesehen werden [20]. Ein Teil dieser Videos dienen formalen Lernprozessen, zahlreiche weitere lösen informelle Lernprozesse aus. Analog zu traditionellen Medien erfordert die Vielzahl an Quellmaterial eine hohe Informationskompetenz hinsichtlich adäquater Such- und Bewertungsstrategien. Noch weiter relativiert in ihrer Bedeutung für die Gesamtheit des Lernens werden dediziert E-LearningSysteme durch das Internet der Dinge. Die Nutzung von Teslas Autopilot bringt kognitives Verständnis und affektive Erfahrungen für Technologien des maschinellen Sehens zum Erkennen der Umgebungsbedingungen und Big-DataAnalysen auf Basis künstlicher Intelligenz in den über eine Million im Echteinsatz befindlichen, voll vernetzten Fahrzeugen.

Eine ähnliche Konzentration auf dedizierte E-LearningTechnologien in der Wirkungsforschung zeigt [5]. Nur $2 \%$ der Konferenzbeiträge beschäftigen sich explizit mit digitalen Medien aus der Alltagsnutzung der Lernenden. Das von [9] für das Lernen als besonders wichtig eingestufte WeChat mit einer Milliarde Benutzern scheint im Bereich der Wirkungsforschung nahezu gar nicht auf.

Die Konzentration auf dedizierte E-Learning-Systeme und das Vernachlässigen von Alltagsmedien, vor allem des Internet der Dinge, erschwert die Verbindung von formalen Lernsettings mit dem dominierenden informellen E-Learning.

\section{Paradigma 3: Immer und überall, Sharing und Personalisieren}

Oftmals wird dieses Paradigma als Vorteil des E-Learning gesehen und als ,je mehr desto besser" maximiert, ohne seine Ambivalenz zu beachten. Immer-und-überall-Lernen bezieht sich auf Orts- und Zeitunabhängigkeit. Dezentralasynchrones Lernen ermöglichen aber auch traditionelle Medien, etwa ein gedrucktes Buch. Der Neuigkeitswert des E-Learning kann also nur in einem speziellen Aspekt liegen. Wesentlich ist hierbei die Kontextualisierung in Sinne des Just-in-Time Learning. Der Lernende erwirbt das Wissen dann, wenn er es benötigt. Ein Beispiel sind umfassende kontextorientierte Hilfefunktionen oder Ad-hoc-Suchen während der Nutzung einer Software, anstelle eines Seminars zum Erlernen möglicherweise in Zukunft gebrauchter Funktionalitäten. Wissen für die Lösung eines konkreten Problems kann aber auch auf Kosten des Verständnisses für seine übergeordneten Ursachen gehen. Sharing ist ein zentrales Wesensmerkmal des konstruktivistisch-kollaborativen Lernens. Aber auch dieses Paradigma sollte nicht un- 
reflektiert maximiert werden. Fundamental neue Gedanken erfordern Rückzugsorte, die das Entstehen von eigenen Ideen und Sichtweisen ermöglichen, um zu fundamentalen Innovationen zu gelangen. Permanentes Sharing kann zum Nivellieren an der gerade vorherrschenden Meinung führen, dient eher dem Hervorbringen inkrementeller Innovationen und kann auf fundamentale Innovationen hemmend wirken ${ }^{1}$.

Ein weiteres Paradigma ist das Personalisieren von Lernformen und Lerninhalten. Auch wenn personalisierte Lernformen aufgrund unterschiedlicher Lerntypen grundsätzlich sinnvoll erscheinen, sollte ein auditiv-visueller Typ in der Lage sein, nicht nur aus Videos zu lernen, sondern auch Texte sinnerfassend lesen zu können. Besonders kritisch ist die uneingeschränkte Personalisierung und Atomisierung von Lerninhalten mittels Technologien des Mikrolernens, wenn sie entsprechend der gerade aktuellen Bedürfnislage der Lernenden erfolgt. Einerseits kann hierdurch die soziale Anschlussfähigkeit verloren gehen. Wenn jeder seine eigene Sprache lernt, kann er sie nur mehr für Selbstgespräche nutzen. Und wenn kaum gemeinsame Bildungsinhalte in einer Kultur vorhanden sind, etwa ein gymnasialer Lesekanon, dann bleiben Gespräche inhaltsleer oder banal. Anderseits sind auch paternalistische Inhalte, solche die von Dritten als sinnvoll für Lernende gesehen werden, durch hohe Personalisierung auf Basis aktueller Bedürfnislagen nicht mehr möglich. Seien es Inhalte, die gesellschaftlich erwünscht sind, deren Notwendigkeit vom Lernenden erst später erkannt wird, oder die für Lernende erst durch Hinführen durch Lehrende interessant werden.

Ein hohes Ausmaß an immer und überall, Sharing und Personalisieren kann zum Nivellieren an der gerade vorherrschenden Meinung führen, fundamentale Innovationen hemmen und reduzieren das Ausmaß gesellschaftlich erwünschter Lerninhalte und sozialer Anschlussfähigkeit.

\section{Paradigma 4: Annahme eines Technikdeterminismus}

Eine häufig anzutreffende implizite Annahme der E-Learning-Forschung ist, dass die eingesetzte Technologie Eigenschaften aufweise, die das Lernen auf eine bestimmte Art deterministisch verändert. Je nach Granularitätsgrad der Aussagen lauten diese dann: E-Learning bewirkt ..., Tablets reduzieren ..., Facebook macht ... Besonders dramatisch zeigt sich ein solcher Technikdeterminismus anhand der Klage der Eltern eines tödlich verunglückten Rennrad-

\footnotetext{
${ }^{1}$ Immanuel Kant publizierte nach seiner Berufung an die Universität Königsberg elf Jahre nahezu nichts, verzichtete also auf Sharing, um eigenständig an fundamentalen Neuerungen zu arbeiten und 1781 mit der Kritik der reinen Vernunft eines der prägendsten Werke der westlichen Philosophiegeschichte vorzulegen. Vgl. hierzu auch die aktuelle wissenschaftstheoretische Kritik am Peer-Review-Verfahren aus der Sicht der Innovationshemmung.
}

fahrers gegenüber der digitalen Plattform Strava². Er wollte den Rekord für einen Streckenabschnitt brechen [19]. Bei diesem Paradigma wird davon ausgegangen, dass bestimmte Auswirkungen der eingesetzten E-Learning-Technologie immanent seien und das Verhalten der Lernenden determinieren. Hierbei wird versucht, über statistisch signifikante Korrelationen Wirkungsmuster von Lerntechnologien zu identifizieren, um Prognosen für Veränderungen bei Lernenden durch ihren Einsatz zu erstellen. Hierbei bleibt offen, wo soziokulturelle Rahmenbedingungen, der Entscheidungsspielraum und letztendlich der freie Wille als zu tiefst menschliche Eigenschaft Platz finden. Ein weiteres Problem ist der Granularitätsgrad der Aussagen: Welche Technologien, welche Formen des E-Learning, Auswirkungen auf welche Lernziele bei welchen Lernenden?

\section{Paradigma 5: Wirkungsforschung mit WEIRD- Probanden}

96\% der Studien, die in führenden Journalen der Verhaltenswissenschaft publiziert werden, sind mit WEIRD-Probanden durchgeführt [12]. Diese stammen aus der Western Hemisphere, sind Educated und kommen aus Ländern die Industrialized, Rich und Developed sind. Meist sind es Bachelorstudierende an US-Eliteuniversitäten. Henrich et al. [12] zeigen, dass WEIRD-Probanden besonders ungeeignet sind, um Schlussfolgerungen auf Verhalten und Motivation der restlichen $88 \%$ der Weltbevölkerung zu ziehen. Arbeiten der digitalen Anthropologie [2] argumentieren, dass ein Kulturuniversalismus bei Aussagen zu den Wirkungen von E-Learning verfehlt ist. Die Analyse der Konferenzbeiträge von [5] zeigt, dass sich kaum ein empirischer Beitrag mit Lernenden beschäftigt, die nicht aus WEIRD-Regionen stammen.

Die Problematik dieses Paradigmas verstärkt sich, wenn es mit normativen Schlussfolgerungen verbunden wird. Welche Bildungsziele, eingebettet in welchem Bildungsideal liegen den Aussagen zu positiven und negativen Auswirkungen zugrunde? Ein Beispiel hierfür sind die häufig anzutreffenden negativen Einschätzungen der sozialen Kontrollfunktion von WeChat in China. Ausgestattet mit einer ethnozentrischen Brille kommt man zu dieser normativen Schlussfolgerung, ohne zu beachten, dass der eigenen Beurteilung das Humboldt'sche Bildungsideal zugrunde liegt. Es ist geprägt vom autonomen Individuum, das Mündigkeit und Individualität durch Vernunftgebrauch erreicht. Dieses Ideal hat wohl genauso viel Existenzberechtigung wie der Konfuzianismus, der das Lernen und

\footnotetext{
${ }^{2}$ Der wohl größte digitale Sportverein der Welt verbindet 35 Mio. Nutzer und beeinflusst das informelle Lernen nicht nur im kognitiven, sondern auch im affektiven und psychomotorischen Bereich.
} 
Technischer

Lernkontext
Personaler und

soziokultureller Lernkontext

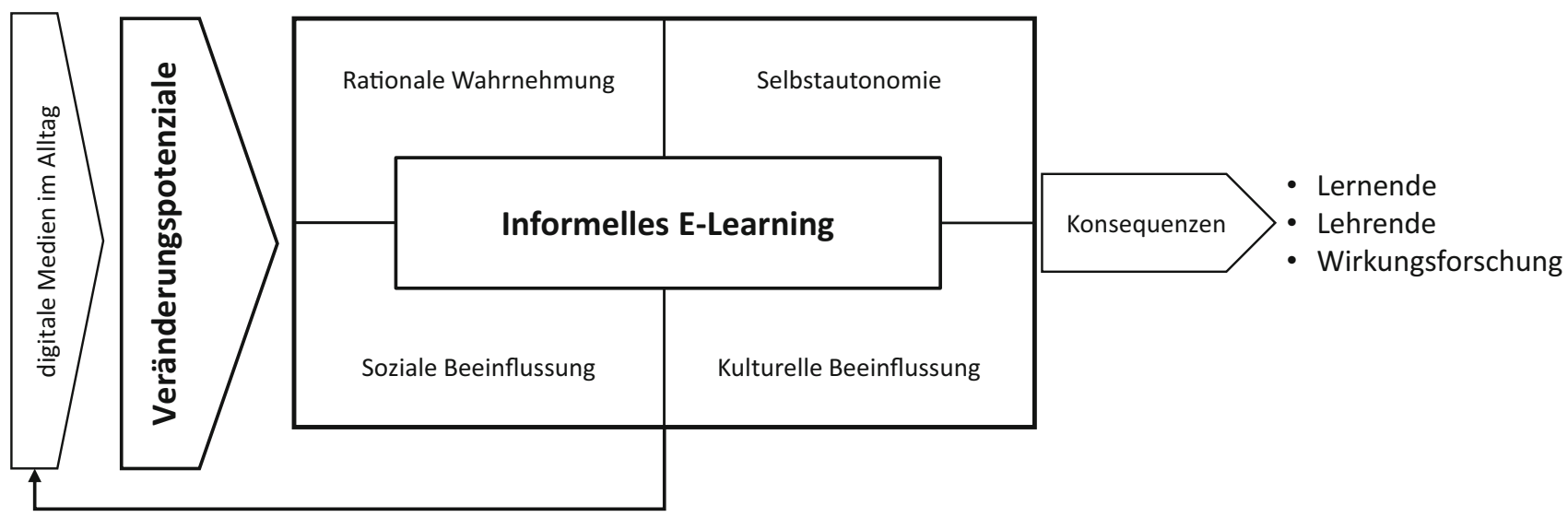

Abb. 1 Ökosystem des informellen E-Learning

Leben in China prägt. Nur ist sein Ideal ein Bürger, der das Gemeinwesen bestmöglich in Harmonie bringt.

\section{Veränderungspotenziale digitaler Medien für das informelle E-Learning}

\section{Ökosystem des informellen E-Learning}

Lehrende sind meist gut vertraut mit dem formal organisierten Bildungssystem an Hochschulen und Schulen. Es umfasst Ziele und Lehrinhalte, Kursformen, Curricula, Organisationseinheiten oder Beurteilungssysteme. Es wird jedoch noch weitgehend isoliert von Veränderungen im Ökosystem des informellen Lernens betrachtet. Die dort stattfindenden Lernprozesse im kognitiven, affektiven und psychomotorischen Bereich sind nicht alternativ, sondern komplementär zum formalen Lernen zu sehen. Ein verstärktes Verständnis für die Veränderungen im informellen Lernen ermöglicht Lehrenden neue Wege in formalen Settings zu gehen, um Lernziele zu erreichen. Seufert et al. [32] sehen in der Verbindung der bisher getrennten Welten von formalem und informellem Lernen eine wesentliche Entwicklungsstufe der digitalen Transformation an Hochschulen.

Das in Abb. 1 dargestellte Ökosystem des informellen E-Learning versucht, sich von den derzeit vorherrschenden Paradigmen im E-Learning zu lösen, um Veränderungen im informellen Bereich des Lernens für formale Settings nutzbar zu machen. Der technische Lernkontext umfasst im Alltag genutzte digitale Medien und ihre Veränderungspotenziale für das informelle E-Learning. Technische Innovationen werden als Potenziale für Veränderungen gesehen und nicht als Determinanten, da sie in unterschiedlichen personalen und soziokulturellen Kontexten zu anderen Wirkungen führen können. Veränderungen in diesen Kon- texten können wiederum zur prioritären Weiterentwicklung bestimmter Technologien und Funktionen führen ${ }^{3}$.

Die Betrachtung von Veränderungspotenzialen als Eigenschaften digitaler Medien ermöglicht zu unterscheiden, ob bestimmte Veränderungen tatsächlich auf technische Innovationen zurückzuführen sind oder aber auf altbekannte Eigenschaften, nun aber substitutiv erbracht durch eine andere Technologie. Ein Beispiel ist orts- und zeitunabhängiges Lernen, das auch durch ein gedrucktes Buch ermöglicht wird. Eine kritische Wirkungsforschung kann sich hierdurch besser auf tatsächliche Neuerungen durch eine spezifische Technologie konzentrieren, anstelle über Dinge zu reflektieren, die unabhängig von dieser Technologie bereits umfassend erforscht werden ${ }^{4}$.

Der personale und soziokulturelle Lernkontext bestimmt, ob eine bestimmte technische Funktionalität wahrgenommen wird, wie und in welchem Ausmaß sie genutzt wird und welche Konsequenzen dies für das informelle E-Learning hat. Wichtige Faktoren der rationale Wahrnehmung sind der wahrgenommene Nutzen hinsichtlich Effizienz, Effektivität und hedonistischer Qualität, die Vereinbarkeit mit persönlichen Werten und Normen sowie vertrauten Lernprozessen, die Komplexität der Nutzung und die Imagewirkung. Dieser Teilbereich wird insbesondere in der Diffusions- und Adoptionsforschung untersucht [28, 29]. Die soziale Beeinflussung basiert auf interpersonellen Netzwerken, die zu kurz- und mittelfristigen Effekten führen. Dieser Teilbereich wird aktuell vor allem im Zusammenhang mit

\footnotetext{
3 „We shape our buildings; thereafter they shape us.“ (Winston Churchill).

${ }^{4}$ Etwa ist die heute oftmals digitalen Medien zugeschriebene Schwächung des Gedächtnisses und der zwischenmenschlichen Kontakte bereits in Platons Phaidros Dialog zu finden - sein Lehrer Sokrates reflektiert über das Medium „Schrift“.
} 


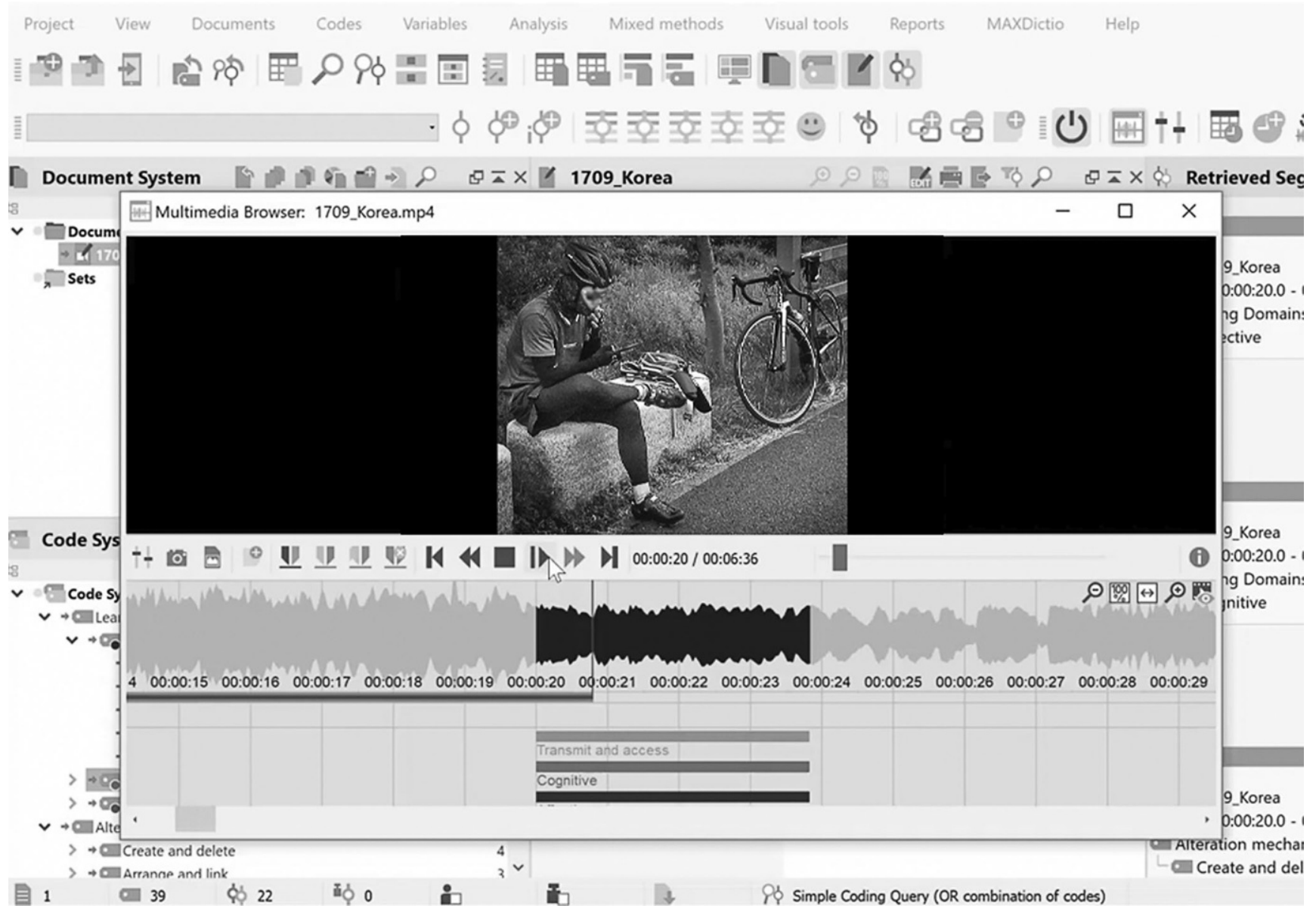

Abb. 2 Analyse einer informellen Lernepisode im affektiven und psychomotorischen Bereich

Sozialen Medien untersucht [34]. Die kulturelle Beeinflussung umfasst fundamentale, langfristige Ausprägungen des Lernkontextes, etwa Ausmaß des Individualismus und der Machtdistanz oder die Langfristorientierung einer Gesellschaft [14].

Die externe Dimension der Selbstautonomie umfasst die Möglichkeit zur Nutzung einer Technologie hinsichtlich Infrastruktur, Kosten und Ausbildung und wird umfassend im Zusammenhang mit „Digital Divide“ diskutiert [29]. Hingegen wird die interne Dimension der Selbstautonomie im Zusammenhang mit E-Learning noch wenig thematisiert. Sie ist die subjektiv wahrgenommene Fähigkeit, sich bewusst für oder gegen eine bestimme Technologie und Nutzungsart zu entscheiden und umfasst in letzter Konsequenz die Frage nach dem freien Willen. Sie wird beispielsweise unmittelbar beeinflusst durch die Werbeausgaben von Unternehmen oder die Berichterstattung in Medien.

\section{Eingesetzte videografische Methode zum Identifizieren von Veränderungspotenzialen}

Ziel der eingesetzten videografischen Methode ist das Erkennen und Beschreiben von Veränderungspotenzialen digitaler Medien für informelle Lernprozesse. Hierbei werden, dem Prinzip der Varianzmaximierung folgend, ungewöhnliche Lernepisoden von Innovatoren und Lead Usern [13] untersucht. Es wird nicht beabsichtigt, eine repräsentative Aussage über das Verhalten einer Grundgesamtheit von Lernenden in Abhängigkeit von bestimmten Formen der Mediennutzung zu treffen. Ein solches Vorgehen würde auf dem oben hinterfragten Paradigma eines Technologiedeterminismus basieren. Vielmehr werden Potenziale und nicht Determinanten identifiziert, die in Abhängigkeit von den in Abb. 1 dargestellten Faktoren zu Veränderungen im informellen Lernen führen können.

Im ersten Schritt werden mit Studierenden im Bachelor- und Masterstudium an der Karl-Franzens-Universität Graz Theorien und Beispiele des informellen Lernens im kognitiven, affektiven und psychomotorischen Bereich besprochen. Im Rahmen der Datenerhebung erstellen die Ler- 
nenden Screencasts von ihrer alltäglichen Nutzung digitaler Medien am Smartphone oder Desktop PC. Hierbei wählen sie solche Nutzungsepisoden aus, die aufgrund der vorangegangenen theoretischen Einführung aus ihrer Sicht informelle Lernprozesse auslösen oder verändern. Bewusst werden diese nicht extra konstruiert, sondern vertraute, alltägliche Lernepisoden aufgezeichnet. Ergänzt werden sie durch Videos und Fotos von informellem E-Learning, das keinen Screencast zulässt, etwa das Steuern einer Drohne. Möglichst zeitnah werden die Lernepisoden durch die Lernenden selbst geschnitten, mit Sprachannotationen zu Nutzungsmotiven und wahrgenommenen Lernprozessen versehen, in interkulturell zusammengesetzten Teams - mit Lernenden aus bis $\mathrm{zu}$ sieben Nationen - diskutiert und auf Basis von Lerntheorien in schriftlichen Arbeiten analysiert. Ein Teil der Lernenden protokolliert ihre gesamte Nutzung digitaler Medien im Alltag innerhalb einer Woche, wobei die Day Reconstruction Method [16] zum Einsatz kommt. Im Zeitraum Oktober 2017 bis Juli 2019 hielten 172 Lernende 543 Lernepisoden in Videoform fest und annotierten sie.

Ergänzend hält der Autor Lernepisoden in entlegenen Regionen und anderen Kulturkreisen fest, um die oben angesprochenen Limitierung auf WEIRD-Lernende zu überwinden. Beispiele sind Dörfer im Himalaya, die zentraliranische Wüste, der Norden Japans, die Japanischen Alpen und Palästina. Hierfür kommen die Methoden der Videografie und des Participatory Action Research [10, 17] zum Einsatz. Um die methodische Anforderung zu erfüllen, bewegte sich der Autor möglichst im natürlichen Umfeld der Lernenden, meist mittels öffentlicher Verkehrsmittel und Langstreckenfahrten mit dem Rad. Zum Schutz der Privatsphäre werden videografisch aufgenommene Personen im Vorhinein um Genehmigung gefragt oder die Lernsequenz wird so aufgenommen bzw. nachträglich bearbeitet, dass ein Identifizieren nicht möglich ist.

Die Datenanalyse für ausgewählte Lernepisoden basiert auf den Prinzipien der Grounded Theory sowie der qualitativen Inhaltsanalyse unter Berücksichtigung umfassender Gütekriterien [3, 18] und erfolgt mittels der Software MAXQDA (VERBI Software - Consult - Sozialforschung $\mathrm{GmbH}$, Berlin, Deutschland). Basierend auf einer Literaturanalyse wird zunächst ein initiales Kategoriensystem der Veränderungspotenziale gebildet. Danach werden einzelne Teilsequenzen ausgewählter Lernepisoden unter Zuhilfenahme der Annotationen im Material codiert, zu Gruppen zusammengefasst und Kategorien zugeordnet. Während des gesamten Analyseprozesses wird das Kategoriensystem iterativ verfeinert und bestmöglich angepasst. Der Analyseprozess wird von drei Teammitgliedern vorgenommen, wobei auf konsistente Codierung und Kategorisierung geachtet wird. Zusätzlich werden mit Lernenden Fokusgruppen durchgeführt, um Lernepisoden im Detail zu diskutieren.
Gegenüber der üblichen Befragung hat Videografie den Vorteil, dass auch informelle, also nebenläufige, Lernprozesse festgehalten werden, derer sich nicht retrospektiv nach einiger Zeit ,erinnert“" werden muss, sondern die unmittelbar oder sehr zeitnah aufgezeichnet und annotiert werden, und das Medium Video auch Informationen festhält, die in Texten oder Logfiles nicht erkennbar sind. Da das aufgenommene Material sehr vielschichtig und facettenreich ist, sind weitere umfassende Analysen geplant. Abb. 2 zeigt die Analyse von informellem E-Learning, das der Autor im Rahmen einer ethnografischen Feldstudie aufzeichnete. Für eine ausführliche Darstellung der Methode siehe [26].

\section{Veränderungspotenziale digitaler Medien für das informelle E-Learning}

\section{Erstellen, Verteilen und Löschen}

Medialisierung ist die Repräsentation eines bestimmten Ausschnitts der physischen Welt mittels Medien. Hierbei wird nicht notwendigerweise beabsichtigt, diese physische Welt möglichst genau und objektiv wiederzugeben. Ein Beispiel sind virtuelle Welten, wie sie im E-Sport zum Einsatz kommen und informelles Lernen in den Bereichen Strategie und Teambuilding auslösen. Medialisierung ist seit der Verbreitung des Buchdrucks, Radios und TV allgegenwärtig. Besonders prägend ist das Veränderungspotenzial Omnipräsenz von Produktionsmitteln für digitale Inhalte. War die Produktion und Verteilung von Videos noch vor wenigen Jahren meist spezialisierten Produktionsfirmen und staatlichen Sendeanstalten vorbehalten, nutzen heute $60 \%$ der Weltbevölkerung Smartphones, die auch ein Produktionsmittel für Videos sind. $92 \%$ leben in einem Gebiet, das mit mobilem Breitband zum Verteilen dieser Videos versorgt ist [15]. Selbsterstellte Videos werden zu einem alltäglichen Kommunikationsmittel, das auch in formalen Settings verstärkt genutzt werden kann. Ein Beispiel ist das Aufzeichnen der Usability von digitalen Medien in der Alltagsnutzung und die anschließende Analyse im Rahmen einer Lehrveranstaltung. Die Omnipräsenz von Kommunikationsnetzen führt zum Veränderungspotenzial Echtzeitreichweite. Der US-amerikanische Präsident Donald Trump erreicht 65 Mio. Follower direkt ohne klassische Medien als Intermediäre und der damit verbundenen Zeitverzögerung und inhaltlichen Veränderung. Alle 1500 Tageszeitungen der USA weisen zusammen eine Auflage von 55 Mio. auf. Dieses Potenzial ermöglicht Lernenden, Informationen direkt von deren Quelle $\mathrm{zu}$ erhalten, ohne Umweg über Medien, Fachaufsätze, Lehrbücher oder Lehrende. Das oft als „Demokratisierung der Massenkommunikation" bezeichnete Potenzial erfordert bei Lernenden eine deutlich höhere Informationskompetenz, um Informationen auswählen und interpretieren $\mathrm{zu}$ können, und 
Tab. 2 Beispiele, wie der Autor Veränderungspotenziale für das informelle E-Learning in der formalen Lehre nutzt

\begin{tabular}{ll}
\hline $\begin{array}{l}\text { Veränderungspotenzial Informelles E-Learning } \\
\text { für das informelle }\end{array}$ & Formales Setting
\end{tabular}

E-Learning

Medialisierung

Omnipräsenz der

Produktionsmittel

für digitalen Con-

tent

Echtzeitreichweite/

Kopierbarkeit/

Unmittelbarkeit

Nachvollziehbarkeit

Analyse der Tracking- und Personalisierungsfunktionen auf alltäglich verwendeten E-Commerce-Sites anhand von Screencasts

Teilbarkeit

En-passant-Nutzung eines Lernmanagementsystems (LMS) am Arbeitsplatz in Form des Mikrolernens

Multiperspektivität

Analyse der Nutzung von digitalen Medien des asiatischen Raums, insbesondere WeChat, Qq.com, QZone und Alibaba durch multikulturell zusammengesetzte Gruppen

Assoziativität

Einsatz von Game-based Learning zur Entdeckung von Verbesserungsmöglichkeiten der Funktionen und Customer Experience von digitalen Medien im Alltag

Durchsuchbarkeit

Eigene Recherche in Medien aus unterschiedlichen Kulturkreisen als ersten Schritt im Inverted Classroom (WeChat, baidu.com, sputniknews.com, chinatoday.com, Twitterfeed des US-Präsidenten) ${ }^{\mathrm{a}}$

\begin{abstract}
Übertragung auf Unternehmensstrategien
Analyse der technischen Funktionalitäten hinsichtlich kognitiver, affektiver und psychomotorischer Auswirkungen
\end{abstract}

Verbindung von drei Hörsälen innerhalb der USA mittels Videolink, Tische mit integrierten PCs für Annotationen der Kursunterlagen und Online-Fragestellungen (Tucson, Arizona 1992-1993)

Erarbeitung der zugrunde liegenden CRM-Funktionalitäten und betriebswirtschaftlichen Ziele der anbietenden Unternehmen

Synchrones Teletutoring und feingranulare digitale Lernunterlagen im LMS (Online MBA, City University of Hong Kong, 1998-1999)

Methodengestützte Analyse der Funktions- und Customer-Experience-Unterschiede ${ }^{\mathrm{a}}$

Methodenbasierte Analyse von Verbesserungsmöglichkeiten (initiiertes und geleitetes dreijähriges $E U$ Projekt zu Game-based Learning in sieben Nationen, 2007-2009)

Methodenbasierte Analyse der Funktionen und Inhalte zur Entwicklung von Schlüsselqualifikationen

ain Planung/Durchführung

bricht Quasi-Informationsmonopole von Medienhäusern, Verlagen und Lehrenden.

Das Veränderungspotenzial Kopierbarkeit ermöglicht die Vervielfältigung von Inhalten ohne Qualitätsverlust und nahezu ohne Grenzkosten. Dies führt zu einer deutlichen Ausweitung des Informationsangebots und zur Veränderung von Geschäftsmodellen, etwa bei Lehrbuchverlagen. Nachvollziehbarkeit führt zum Generieren von Verhaltensdaten als Beiprodukt jeglicher Aktivitäten mit digitalen Medien, die wiederum das Informationsverhalten von Lernenden beeinflussen können, etwa durch „Meist gelesen“Rubriken. Dies wird oftmals im Zusammenhang mit dem Begriff der „Echokammer“ thematisiert, wobei zu hinterfragen ist, welche Veränderungen hier insbesondere Soziale Medien tatsächlich bringen und was altbekannte Effekte sind, etwa durch den Besuch einer Universität, die eine bestimmte Zusammensetzung von Lehrenden und Lernenden hat. Das Potenzial des nicht zweifelsfreien Löschens, also der Möglichkeit der Existenz von weiteren Kopien, gestattet Lernenden den Zugriff auf andernfalls nicht mehr verfügbare Informationen, kann aber auch die Bereitschaft senken, eigene Informationen zu teilen.

\section{Anordnen und Verlinken}

Durch das Potenzial Teilbarkeit wird ermöglicht, Informationen und Kommunikationsinhalte in einzelne Pakete aufzusplitten, neu zu arrangieren und mit anderen $\mathrm{zu}$ teilen. Dies besitzt vor allem im Bereich des Mikrolernens besondere Bedeutung. Multiperspektivität erlaubt dem Lernenden, einen bestimmten Sachverhalt auf Basis unterschiedlicher Informationsquellen zu beurteilen. Assoziativität beruht auf nichtlinearen Bewegungspfaden innerhalb eines grundsätzlich nicht abgeschlossenen Informationsund Kommunikationsraums. Der Lernende geniert durch sein Assoziationsverhalten ein eigenes, individuelles und personalisiertes Informationsangebot. Diese Veränderungspotenziale eröffnen neue Möglichkeiten, sich im Sinne eines konstruktivistischen Lernens selbst ein Bild von einem Sachverhalt zu machen und tief in ein Themengebiet einzudringen, nicht limitiert durch einige wenige Informationsquellen und Kommunikationspartner. Gleichzeitig können sie auch genau das Gegenteil, seichtes Lernen, bewirken: durch immer kleinere Informationspakete und Kommunikationsinhalte bei gleichzeitiger Reduzierung der Pausen zur Reflexion innerhalb des Informationsstroms. 


\section{Zugriff}

Durch das Veränderungspotenzial Unmittelbarkeit erwarten Lernende zunehmend möglichst aktuelle Informationen und holen sich diese direkt bei der Quelle. Gleichzeitig sinkt die Fähigkeit zum Verzicht auf unmittelbare Befriedigung eines Informationsbedürfnisses. Findet ein Lernender eine bestimmte Information nicht gleich oder kann sie nicht unmittelbar inhaltlich erschließen, wird weitergesurft, werden knappe Fragen an Peers gestellt. Das für konstruktivistisches Lernen wichtige Austauschen von Meinungen ersetzt das mühsame Studieren von Inhalten, auch wenn dies bei Fakten- und Methodenwissen nach wie vor notwendig ist. Dies kann zum oben angeführten seichten Lernen führen, einer der größten Dysfunktionalitäten des informellen E-Learnings.

Durchsuchbarkeit bedeutet, dass Lernende selbst in einem nahezu unbegrenzten Informationsangebot suchen und Auswahlentscheidungen treffen können. Sie sind weit weniger auf vorgefertigte Auswahlstrategien, etwas durch Bibliothekskataloge, Zeitungsredaktionen oder Lehrende angewiesen. Diese Suchergebnisse offenbaren jedoch nicht den in den Suchergebnissen referenzierten Inhalten immanente Eigenschaften, um etwa eine richtige Antwort auf eine Lernfrage zu liefern, sondern sind von der Suchmaschine ad hoc neu generierter Inhalt. Das Gefundene hängt vom Algorithmus der Suchmaschine und seiner zufälligen oder beabsichtigten Interpretation des Suchbegriffes, der Selektion der Inhalte sowie deren Präsentation ab. Somit sind Suchmaschinen nicht ,neutrale“ Werkzeuge, um Informationen zu finden, sondern Inhaltsproduzenten mit ihren eigenen Intentionen - analog zu traditionellen Medien. Gleichzeitig generiert der Lernende durch individuelle Suchanfragen und sein assoziatives Weiterverzweigen aufgrund der Ergebnisse sein eigenes Informationsangebot. Dies führt nicht nur zu einem deutlich erweiterten Raum für informelles E-Learning, sondern auch zu einer starken Heterogenisierung der Informationsbasis der Lernenden untereinander, aber auch im Verhältnis zum Lehrenden.

\section{Ansätze für einen Paradigmenwechsel}

\section{Informelles E-Learning für formale Lehre nutzen}

Informelles E-Learning findet statt, unabhängig, ob Lehrende es nutzen, beeinflussen oder ignorieren. Es kann von Lehrenden als wichtiger Verbündeter zur Erreichung von Lernzielen genutzt werden. E-Sport trägt nicht nur die Schuld an schlecht ausgeschlafenen und unkonzentrierten Lernenden, sondern kann auch zu einem Verständnis für strategisches Verhalten und Teambuilding sowie zu Selbstdisziplin, Neugierde und Ehrgeiz führen. Ein vom Autor in einem CRM-Kurs umgesetztes Beispiel ist die kritische Analyse von Beeinflussungsstrategien auf E-CommerceSites, die zu affektiven Lernprozessen der Nutzer führen, etwa Wahrnehmung ausgewählter Produkte, Markentreue und reduzierte Preissensibilität. Der Methode des Inverted Classroom [11] folgend erschließen die Lernenden zunächst selbstständig Inhalte durch das Studium theoretischer Grundlagen zu Beeinflussungsstrategien und erstellen annotierte Screencasts ihres alltäglichen Nutzungsverhaltens von E-Commerce-Sites. Danach erfolgt in formalen Settings die kritische Auseinandersetzung mit den festgestellten Beeinflussungsstrategien auf Basis softwaretechnischer Funktionen wie Profiling, Collaborative Filtering und Behavioral Targeting. Weitere vom Autor umgesetzte Beispiele, die informelles E-Learning in der formalen Lehre nutzen, zeigt Tab. 2.

\section{Schlüsselqualifikationen vermitteln}

Veränderungspotenziale für das informelle E-Learning besitzen einen deutlich ambivalenten Charakter. Sie können funktional oder dysfunktional auf Lernziele wirken. Entscheidend hierfür ist die konkrete Ausgestaltung der Nutzung, wobei der Schlüsselqualifikation Informationskompetenz eine besondere Bedeutung zukommen. Technische Fertigkeiten zum Zugriff auf unterschiedliche Informationsquellen sind hierbei nur ein Aspekt. Wie oben beim Veränderungspotenzial „Erstellen, Verteilen und Löschen“ besprochen, nutzen Lernende immer mehr Informationsquellen und überspringen klassische Vermittlungsinstanzen wie ,ihre“ Tageszeitung, das staatliche Fernsehprogramm, Buchverlage, Lehrer oder Eltern, um sich Informationen direkt vom Ort des Entstehens zu holen. Um Aussagen des US-amerikanischen Präsidenten für eine Seminararbeit zu nutzen, sind sie nicht mehr auf den Bericht von einer Pressekonferenz in einer Qualitätszeitung angewiesen, sondern erhalten sie zeitgleich mit Journalisten im Twitterfeed des Präsidenten. Gleichzeitig verteilen Lernende Informationen an ihre Peers und die Öffentlichkeit, was in der Vergangenheit riesige Druckmaschinen oder Sendemonopole erforderte. Sie werden somit auch zum umfassenden Produzenten und Distributor von Informationen. Damit diese Möglichkeiten hinsichtlich formaler Lernziele, aber auch einer liberalen Demokratie funktional wirken, sind wesentliche Kompetenzen notwendig: Bewerten der Relevanz und Glaubwürdigkeit der Quelle und ihrer Information, Erkennen von nicht offensichtlichen Zusammenhängen, Interpretieren vor einem spezifischen soziokulturellen Hintergrund (das Social-Credit-System von WeChat nicht ohne Kenntnis des Konfuzianismus in China beurteilen), Einordnen in einen Gesamtzusammenhang, Schaffen eines Mehrwerts durch eigenständiges Denken sowie Verantwortung für das eigene Tun und die Auswirkungen durch das Verteilen der Infor- 
mationen. Oder: Schlüsselqualifikationen erwerben, die bisher Qualitätsjournalistinnen und -journalisten vorbehalten waren.

\section{Dedizierte E-Learning-Systeme mit ubiquitären Alltagsmedien, auch dem Internet der Dinge, verbinden}

Dedizierte E-Learning-Systeme erlauben die Vorgabe einer Lernstruktur, gesicherte Informationen und kontrollierte Methodenvielfalt. Um die derzeit meist noch getrennten Ökosysteme von formalem und informellen Lernen $\mathrm{zu}$ verbinden, sollten jene Technologien, die den Lernenden aus ihrem Alltag vertraut sind, mit dem E-Learning-System für das formale Setting verbunden werden. Dies erlaubt hinsichtlich Daten, Funktionen und Benutzungslogik einen einheitlichen Workflow im gesamten E-Learning-Ökosystem. Ein Beispiel ist das Uploading von Videosequenzen, die mit dem Smartphone erstellt wurden oder aus dem eigenen Twitter Feed stammen, um sie in formalen Settings zu analysieren. Besonders wertvoll hierfür können Daten aus dem Internet der Dinge sein. Gerade sie geben Aufschluss über informelle, also unbewusste Lernprozesse.

\section{Kontextspezifische Feldforschung intensivieren}

Die Wirkungsforschung im Bereich E-Learning konzentriert sich auf den Wissenstransfer in formalen Settings, wird überwiegend mit Lernenden aus WEIRD-Ländern durchgeführt und zeigt eine Konzentration auf kognitives E-Learning, während der affektive und insbesondere der psychomotorische Bereich ${ }^{5}$ weitgehend unerforscht sind. Damit ist nur ein kleiner Teil des E-Learning-Ökosystems abgedeckt und gleichzeitig wird von einer sehr speziellen Probandengruppe auf allgemeine Wirkungen des E-Learning geschlossen. Die im vorliegenden Beitrag dargestellte videografische Feldforschung umfasst neben dem kognitiven, auch das affektive und psychomotorische E-Learning und führt sie auch in Kulturkreisen durch, die in der E-Learning-Forschung weitgehend unberücksichtigt bleiben. Beispiele sind indische Himalaya-Regionen, entlegene Gebiete im Norden Japans und den Japanischen Alpen mit gänzlich anderem Nutzungsverhalten als in Tokyo, Dörfer in der iranischen Wüste und Schulkinder in afrikanischen Bergdörfern. Besonders hervorzuheben ist, dass gerade das informelle E-Learning durch seinen nebenläufigen, oftmals unbewussten Charakter nur unzureichend durch retrospektive Befragungen erforscht werden kann. Gleichzeitig muss angemerkt werden, dass diese Methode einen hohen zeit-

\footnotetext{
5 Vgl. hierzu [26] zum Einsatz von Self-Measurement-Devices in in-
} formellen Lernepisoden. lichen und persönlichen Einsatz der Forscherinnen und Forscher erfordert.

\section{Verständnis für informelles E-Learning bei Lehrenden entwickeln}

Ein grundlegendes Verständnis der Lehrenden für informelles E-Learning ist Voraussetzung, um die angeführten Ansatzpunkte für einen Paradigmenwechsel nutzen zu können. Es ist wohl zumindest ebenso wichtig wie Weiterbildung in technischen Fertigkeiten oder methodischen Ansätzen. Entscheidend sind nachhaltig geänderte Tiefenstrukturen des informellen Lernens, weniger reine Substitutionseffekte oder Moden. Die Frage, ob das Mitschreiben auf Tablets eher zur Erreichung von Lernzielen führt als jenes auf Papier, scheint nebensächlich im Vergleich zu nachhaltig geänderter sozialer Interaktion durch E-Sport, jenem Interaktionsraum, der traditionelle Soziale Medien in vielen Bereichen ablöst. Die Bandbreite der Veränderungen bei Lernenden reicht bis zu anderen Formen der Wahrnehmung und des Lernens aufgrund der neuronalen Plastizität der Gehirnstrukturen nach 40 Jahren der Digitalisierung des Alltags und der damit einhergehenden geänderten biologischen Hardware [25].

Mithilfe dieses Verständnisses können Lehrende ihre Basisstrategie entwerfen. Eine davon ist, ihren Rückstand gegenüber der Nutzung digitaler Medien durch Lernende hard- und softwarezentriert aufzuholen und diese Nutzung zu imitieren. Sie stellt den derzeitigen Mainstream in Form von Tablet-Klassen oder dem omnipräsenten PowerPoint dar. Wenn dies ohne Berücksichtigung von Lernzielen, personalen und soziokulturellen Kontextfaktoren und den individuellen Stärken des Lehrenden erfolgt, ist der Nutzen fraglich, bestenfalls jedoch ein Zufallstreffer. Eine andere Strategie besteht darin, ganz bewusst nicht die alltägliche Mediennutzung der Lernenden zu kopieren, sondern $\mathrm{zu}$ ihr komplementär zu sein. Hierbei werden besonders Kompetenzen gefördert, die durch informelles E-Learning erodieren können: sinnerfassend lesen, zuhören, wesentliche Punkte erkennen und notieren, insbesondere auch die Konzentrationsfähigkeit in Form des Monotasking. Dann kann es auch sein, dass Vorlesungen, in denen es Studierenden nicht erlaubt ist, elektronische Geräte $\mathrm{zu}$ nutzen, in YouTube hunderttausende Abonnenten haben [33]. Eine dritte, oft unbewusst gewählte Strategie ist, informelles E-Learning als wesentlichen Teil des Lern-Ökosystems zu ignorieren.

\section{Resümee}

Die Nutzung digitaler Medien an Hochschulen ist wohl erst ganz am Anfang. Nicht, dass im formalen Lernen zu we- 
nig davon eingesetzt wird. Zur Unterstützung des Wissenstransfers vom Lehrenden zu Lernenden gehören Lernmanagementsysteme, MOOCs und auf Video aufgezeichnete Vorlesungen weitgehend zum Alltag. Mehr als die Hälfte der Lehrenden nutzt sie regelmäßig, nahezu alle gelegentlich. Der Großteil der Beeinflussung des Lernens durch digitale Medien erfolgt aber außerhalb des Hörsaal - im Alltag der Lernenden. Nicht nur durch YouTube und Wikipedia, auch durch E-Sport und Vitalsensoren im Internet der Dinge. Dieses informelle E-Learning bleibt in formalen Lernsettings noch weitgehend ungenutzt und scheint in der E-Learning-Forschung kaum auf. Der vorliegende Beitrag versucht Lernende und Lehrende zu ermuntern, eine etwas andere Sicht auf E-Learning einzunehmen, losgelöst von jeglichem Technikdeterminismus in Form von Wenn-DannAussagen. Vielmehr steht die, zumindest partiell, autonome menschliche Entscheidung im Mittelpunkt - wunderbar in ihren Möglichkeiten erweitert durch digitale Medien mit den hier analysierten Veränderungspotenzialen. Bildung bleibt aber wohl weiterhin ein mühsamer menschlicher und somit sozialer Prozess, sieht man von nicht weiter thematisierten neurochirurgischen Eingriffen, gentechnologischen Maßnahmen und IoT-basierten Gehirnimplantaten ab. Das kann auch eine gute Nachricht sein. Für alles andere haben wir Rechner mit ihren Algorithmen.

Funding Open access funding provided by University of Graz.

Open Access Dieser Artikel wird unter der Creative Commons Namensnennung 4.0 International Lizenz veröffentlicht, welche die Nutzung, Vervielfältigung, Bearbeitung, Verbreitung und Wiedergabe in jeglichem Medium und Format erlaubt, sofern Sie den/die ursprünglichen Autor(en) und die Quelle ordnungsgemäß nennen, einen Link zur Creative Commons Lizenz beifügen und angeben, ob Änderungen vorgenommen wurden.

Die in diesem Artikel enthaltenen Bilder und sonstiges Drittmaterial unterliegen ebenfalls der genannten Creative Commons Lizenz, sofern sich aus der Abbildungslegende nichts anderes ergibt. Sofern das betreffende Material nicht unter der genannten Creative Commons Lizenz steht und die betreffende Handlung nicht nach gesetzlichen Vorschriften erlaubt ist, ist für die oben aufgeführten Weiterverwendungen des Materials die Einwilligung des jeweiligen Rechteinhabers einzuholen.

Weitere Details zur Lizenz entnehmen Sie bitte der Lizenzinformation auf http://creativecommons.org/licenses/by/4.0/deed.de.

\section{Literatur}

1. Bratengeyer E, Steinbacher P, Martina F, Neuböck K, Kopp M, Gröblinger O, Ebner M (2016) Die österreichische Hochschul-ELearning-Landschaft. BoD

2. Budka P, Kremser M (2004) CyberAnthropology—anthropology of cyberculture. In: Khittel S, Plankensteiner B, Six-Hohenbalken M (Hrsg) Contemporary issues in socio-cultural anthropology: perspectives and research activities from Austria. Loecker, Wien, S 213-226

3. Charmaz K (2014) Constructing grounded theory: a practical guide through qualitative analysis. SAGE, London
4. Dohmen G (2001) Das informelle Lernen. Bundesministerium für Bildung und Forschung (BMBF), Bonn

5. Findenig L (in Arbeit) Masterarbeit am Institut für Informationswissenschaft und Wirtschaftsinformatik. Karl-Franzens-Universität Graz, Graz

6. Frees B, Koch W (2018) ARD/ZDF-Onlinestudie 2018: Zuwachs bei medialer Internetnutzung und Kommunikation. Media Perspekt 9:398-413

7. v. Glasersfeld E (1995) A constructivist approach to teaching. In: Steffe LP, Gale J (Hrsg) Constructivism in education. Erlbaum, Hilldale, S 3-15

8. Harasim L (2017) Learning theory and online technologies. Taylor and Francis, London

9. Hart J (2019) Top tools for learning. Center for learning \& performance technologies. www.toptools4learning.com. Zugegriffen: 14. Okt. 2019

10. Heath C, Hindsmarsh J, Luff P (2010) Video in qualitative research. SAGE, London

11. Handke J, Sperl A (2012) Das Inverted Classroom Model. Begleitband zur ersten deutschen ICM-Konferenz. Oldenburg, München

12. Henrich J, Heine SJ, Norenzayan A (2010) The weirdest people in the world? Behav Brain Sci 33(2/3):61-83

13. v. Hippel E (1986) Lead users. A source of novel product concepts. Manage Sci 32:791-805

14. Hofstede G, Hofstede GJ, Minkov M (2010) Cultures and organizations: software of the mind. McGraw-Hill, New York

15. International Telecommunication Union (2019) ICT facts and figures. ITU, Genf

16. Kahneman D, Krueger AB, Schkade DA, Schwarz N, Stone AA (2004) A survey method for characterizing daily life experience: the day re-construction method. Science 306(5702):1776-1780

17. Kindon S, Pain R, Kesby M (2007) Participatory action research: origins, approaches and methods. In: Kindon S, Pain R, Kesby M (Hrsg) Participatory action research approaches and methods. Routledge, London, New York

18. Kuckartz U (2012) Qualitative Inhaltsanalyse. Methoden, Praxis. Computerunterstützung. Beltz Juventa, Weinheim, Basel

19. Lindsey J (2012) App drove biker to greater speed, but didn't cause his death. WIRED 06/25/12. https://www.wired.com/2012/ 06/opinion-strava-lawsuit. Zugegriffen: 12. Sept. 2019

20. MerchDope (2018) 37 mind blowing youtube facts, figures and statistics-2018. https://merchdope.com/youtube-statistics. Zugegriffen: 26. Mai 2018

21. MMB-Institut für Medien- und Kompetenzforschung (2017) Welche der folgenden Technologien und Anwendungen nutzen Sie bzw. setzen Sie ein? Statista. https://de.statista.com/statistik/daten/ studie/733670/umfrage/nutzung-von-lerntechnologien-durchhochschullehrende-in-deutschland/. Zugegriffen: 11. Okt. 2019

22. OECD (2019) Education at a glance 2019: OECD indicators. OECD Publishing, Paris

23. Petrovic O, Brand A (Hrsg) (2009) Serious games on the move. Springer, Wien

24. Petrovic O (2017) Wie das Internet der Dinge die Kommunikation von Unternehmen verändert. Wirtschaftsinform Manag 4:68-73

25. Petrovic, O. (2018): Lesen ist unmittelbares Glück. Neue Zürcher Zeitung, 27. Febr. 2018.

26. Petrovic O (2019) Digital media and informal learning: alteration mechanism and captured episodes. In: McLaren BM et al (Hrsg) CSEDU 2018-Revised Selected Best Papers. Springer, Switzerland

27. Pietraß M (2016) Informelles Lernen in der Medienpädagogik. In: Rohs M (Hrsg) Handbuch Informelles Lernen. Springer, Wiesbaden, S 123-142

28. Rogers EM (2003) Diffusion of innovation. Simon and Schuster, New York 
29. Scheerder A, van Deursen A, van Dijk J (2017) Determinants of Internet skills, uses and outcomes. A systematic review of the secondand third-level digital divide. Telemat Inform 34:1607-1624

30. Schmid U, Goertz L, Radomski S, Thom S, Behrens J (2017) Monitor Digitale Bildung: Die Hochschule im digitalen Zeitalter. Bertelsmann Stiftung, Gütersloh

31. Schroeder R (2018) Towards a theory of digital media. Information Commun Soc 21(3):323-339

32. Seufert S, Guggemos J, Moser L (2019) Digitale Transformation an Hochschulen: auf dem Weg zu offenen Ökosystemen. Z Hochschulentwickl 14:85-107

33. Shirky, C. (2014): Da könnte ich auch gleich Ghettoblaster im Seminar erlauben, ZEIT Campus, 15. Oktober 2014, übersetzter Auszug aus medium.com.
34. Song J, Drennan J, Sawang S, Andrews L (2015) Same but different? Mobile technology adoption in China. Inf Technol People 28(1):107-132

Otto Petrovic ist Universitätsprofessor an der Karl-Franzens-Universität Graz. Er arbeitet seit mehr als zwanzig Jahren mit innovativen ELearning-Technologien und erforscht deren Wirkung in verschiedenen Kulturkreisen. Gastprofessuren in Tucson/Arizona, Eindhoven, Hongkong, Essen, Seoul und Tokyo, Berufungen an vier Universitäten, Leitung mehrerer EU-Forschungsprojekte, Mitglied der Telekom-ControlKommission der Republik Österreich, Entwicklung von Strategieplänen für den Eintritt in die Informationsgesellschaft in Deutschland und Österreich, Jurymitglied zahlreicher Innovationspreise. 